\title{
NuWro - Monte Carlo generator of neutrino interactions
}

\author{
Jan SOBCZYK* \\ Wroclaw University \\ E-mail: jsobczyk@ift.uni.wroc.pl
}

Performance of the hadronization model of NuWro Monte Carlo generator of neutrino interactions is discussed and confronted with experimental data for charged hadron multiplicities in forward and backward directions. Both neutrino and antineutrino charged current reactions are considered. For neutrino interactions a comparison with GENIE is presented.

10th International Workshop on Neutrino Factories, Super beams and Beta beams June 30 - July 52008

Valencia, Spain

*Speaker. 


\section{Contents}

NuWro is the Monte Carlo generator of neutrino interactions constructed by a group of physicists (Cezary Juszczak, Jarosław Nowak, JTS and also Krzysztof Graczyk) from the Wrocław University during last $\sim 5$ years. The work on NuWro originated from the interest in the performance of the NUX+ FLUKA code in the single pion production region. Currently NuWro serves as a tool to investigate relevance of various theoretical models included (or not) in experimental analysis.

NuWro's basic algorithms follow better known codes (NEUT, NUANCE, NEUGEN/GENIE). In order to facilitate comparisons NuWro allows running simulations choosing easily values of parameters, sets of form-factors, models of nucleus etc.

The distinguished features of NuWro are: fine hadronization model, description of resonance region without Rein-Sehgal approach (the motivation comes from the quark-hadron duality) and effective implementation of spectral function [1] in order to describe correctly distribution of nucleon momenta and binding energies in the impulse approximation scheme.

In the NuWro hadronization model PYTHIA fragmentation routines are used to produce final states for the values of invariant hadronic mass down to $1210 \mathrm{MeV}$. Its performance has to be confronted carefully with available experimental data. The basic observables are overall charged hadron multiplicities and comparisons with NuWro has been presented elsewhere [2]. In this contribution we present more detail investigation of the production of charged hadrons in forward and backward directions.

In Fig 1, first two columns, NuWro predictions and data for neutrino CC reaction on proton and neutron are shown. We present also the predictions of GENIE generator [3]. In the upper row the overall multiplicities are shown while in the middle and bottom row forward and backward contributions are presented separately.

We see that there is good agreement between NuWro and GENIE for $W^{2}>10 \mathrm{GeV}^{2}$. In the case of GENIE non-physical bulges (especially for $v p$ reaction) appear for $W^{2} \in(2.5,10) \mathrm{GeV}^{2}$ which presumably originate from the procedures to combine together PYTHIA and KNO based hadronization models. NuWro does not use KNO arguments and its predictions are smooth.

For proton the data comes from hydrogen and deuterium target experiments. The data points from [4] differ a lot from the others for overall and forward direction multiplicities.

In the case of $v n$ reaction there are large discrepancies between data points for forward and backward hemispheres. Both NuWro and GENIE reproduce well overall multiplicities

In Fig 1, last two columns, NuWro predictions and data for anti-neutrino CC reaction on proton and neutron are shown. For antineutrino reactions we do not have GENIE predictions. For $\bar{v} p$ reaction there are two consistent sets of data. In both reactions (antineutrino on proton and on neutron) NuWro underestimates forward and overestimates backward production while the overall charged hadron multiplicity is reproduced well.

Acknowledgments: The author was supported by MNiSW grants: 3735/H03/2006/31 and 35/N-T2K/2007/0. 


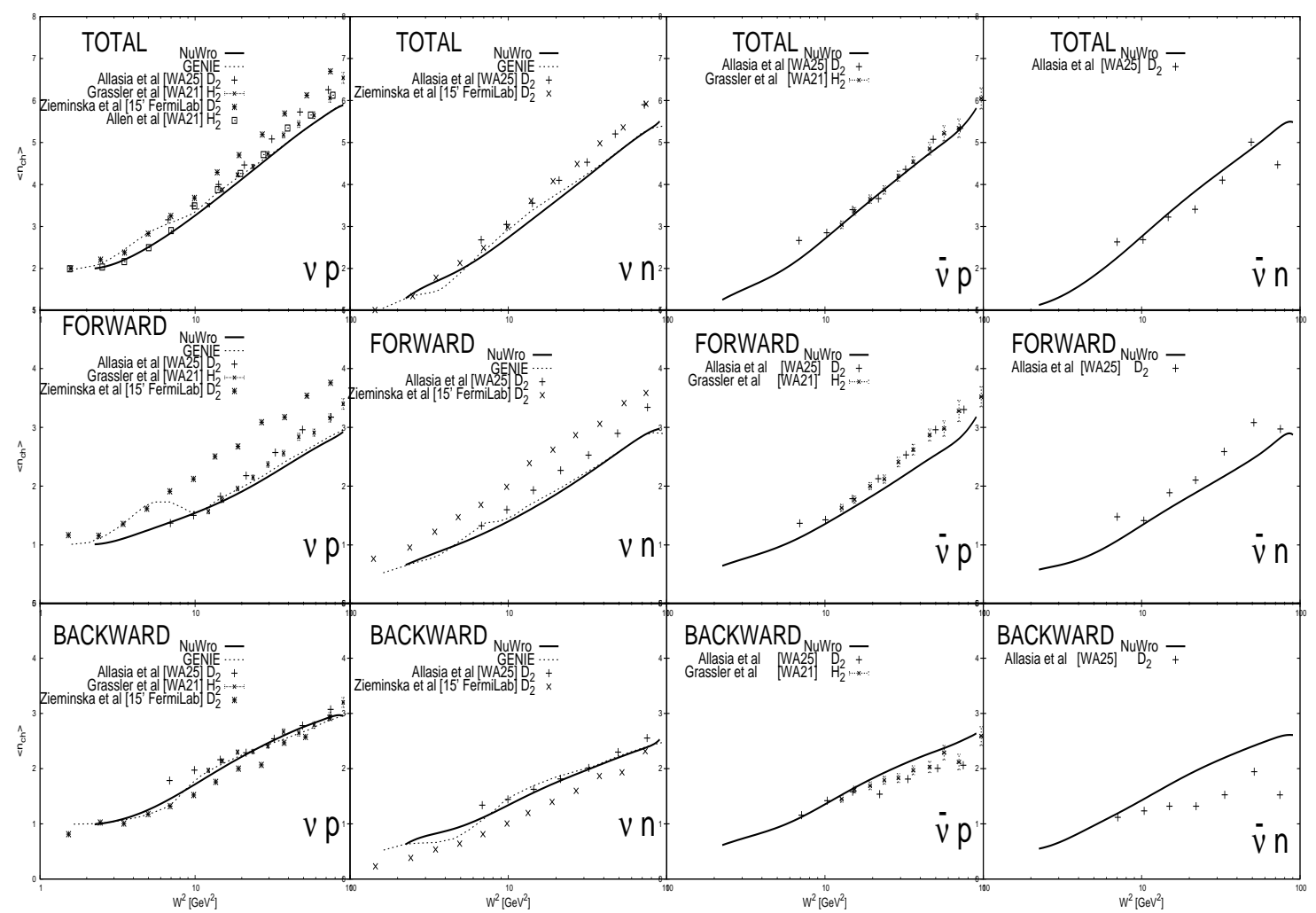

Figure 1: Charged hadron multiplicities for neutrino (first two columns) and antineutrino charged current reactions on proton and neutron. In the first row overall multiplicities are shown and below results for forward and backward directions are presented separately. GENIE predictions are scanned from [3]. Experimental points are taken from [4-7].

\section{References}

[1] A. M. Ankowski, J. T. Sobczyk, Argon spectral function and neutrino interactions, Phys. Rev. C74 (2006) 054316.

[2] J. A. Nowak, Wrocław neutrino event generator, Phys. Scr. T127 (2006) 70.

[3] T. Yang, C. Andreopoulos, H. Gallagher, P. Kehayias, A hadronization model for the MINOS experiment, AIP Conf.Proc. 967 (2007) 269.

[4] D. Zieminska et al Charged-particle multiplicity istribution in vn and vp charged-current interactions, Phys. Rev. D27 (1983) 47.

[5] H. Grässler et al, Multiplicities of secondary hadrons produced in neutrino $p$ and anti-neutrino $p$ charged current interactions, Nucl. Phys. B223 (1983) 269

[6] D. Allasia et al, Fragmentation In Neutrino And Anti-Neutrinos Charged Current Interactions On Proton And Neutron, Z. Phys. C24 (1984) 119.

[7] P. Allen et al, Multiplicity Distributions In Neutrino - Hydrogen Interactions, Nucl. Phys. B181 (1981) 385 . 